\title{
$\mathrm{SC}$

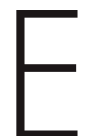

\section{as a regulator of poliovirus and enterovirus 71 infection}

Received: 11 August 2016 Accepted: 08 December 2016

Published: 1 March 2017

\section{Nichole Lynn Orr-Burks ${ }^{1, *}$, Byoung-Shik Shim ${ }^{1, *}$, Weilin $\mathrm{Wu}^{1, *}$, Abhijeet A. Bakre ${ }^{1}$, Jon Karpilow ${ }^{2} \&$ Ralph A. Tripp ${ }^{1}$}

MicroRNAs (miRNAs) regulate virus replication through multiple mechanisms. Poliovirus causes a highly debilitating disease and though global efforts to eradicate polio have sharply decreased polio incidence, unfortunately three countries (Afghanistan, Nigeria and Pakistan) remain polio-endemic. We hypothesize that understanding the host factors involved in polio replication will identify novel prophylactic and therapeutic targets against polio and related viruses. In this data set, employing genome wide screens of miRNA mimics and inhibitors, we identified miRNAs which significantly suppressed polio replication. Specifically, miR-134 regulates poliovirus replication via modulation of ras-related nuclear protein (RAN), an important component of the nuclear transport system. MiR-134 also inhibited other Picornaviridae viruses including EV71, a growing concern and a high priority for vaccination in Asian countries like China. These findings demonstrate a novel mechanism for miRNA regulation of poliovirus and other Picornaviridae viruses in host cells, and thereby may provide a novel approach in combating infection and a potential approach for the development of anti-Picornaviridae strategies.

\begin{tabular}{|l|l|}
\hline Design Type(s) & screening campaign • microRNA profiling by array design \\
\hline Measurement Type(s) & viral genome replication \\
\hline Technology Type(s) & virus plaque assay \\
\hline Factor Type(s) & miRNA $\bullet$ virus \\
\hline Sample Characteristic(s) & immortal human epithelial cell line cell \\
\hline
\end{tabular}

${ }^{1}$ Department of Infectious Diseases, College of Veterinary Medicine, University of Georgia, Athens, Georgia 30602, USA. ²Proventus Bio, 220 Riverbend Rd, Athens, Georgia 30602, USA. *These authors contributed equally to this work. Correspondence and requests for materials should be addressed to R.A.T. (email: ratripp@uga.edu). 


\section{Background and Summary}

Poliovirus (PV), a non-enveloped human enterovirus virus of the Picornaviridae family is the etiological agent of poliomyelitis (polio). Poliovirus symptoms include fever, fatigue, headache, limb pain and permanent paralysis in some cases. Global vaccination campaigns utilizing live-attenuated oral polio vaccines have reduced PV in all but three countries (Afghanistan, Nigeria and Pakistan) which still remain PV-endemic. The global use of live-attenuated vaccines has an increased risk of reversion to wild type and shedding by immune-deficient individuals ${ }^{1,2}$. Though vaccination is effective, treatment of polio is symptomatic. Enterovirus 71 (EV71), a relative of PV, is a major public health burden in the Western Pacific region associated with severe neurologic symptoms of hand, foot and mouth disease (HFMD), a disease most often contracted by children $<5$ years old $^{3}$. More than 7 million cases of HFMD were reported in mainland China from 2008-2012. EV71 vaccination programs are challenged by high cost and vaccine availability ${ }^{4}$. Understanding host gene networks that regulate PV and/or EV71 replication, and development of agents against these targets is a promising strategy to prevent and treat PV while eliminating the issues of reversion and shedding.

RNA interference (RNAi) is an evolutionarily conserved pathway of post transcriptional gene regulation involving small double-stranded non-coding RNAs, microRNAs (miRNAs). The 'guide' strand contains a $6 \mathrm{nt}$ 'seed' region that binds complementary miRNA recognition elements (MRE) within the $3^{\prime}$ untranslated regions (3'-UTR) of transcripts in a RNA induced silencing complex (RISC) causing degradation of transcripts or blocking gene translation ${ }^{5,6}$. PV and EV71 can modulate multiple host pathways including RNAi for their own replication ${ }^{7-13}$. The role of miRNAs in these processes is incompletely understood. To understand the role of miRNAs in viral replication, we undertook a highthroughput screen utilizing miRNA inhibitors and mimics to determine which miRNAs alter the replication of poliovirus and the related EV71.

HEp-2C cells were reverse-transfected with non-targeting siRNA, miRNA negative controls, or gene specific mimics for $48 \mathrm{~h}$. The gene specific mimics corresponded to 1,208 known miRNAs in the human genome. Cytotoxicity was evaluated via CytoTox-Glo assay (Promega). Mimics which were not cytotoxic were evaluated. Transfected cells were either mock infected (no virus) or infected with Sabin-2 strains of PV (MOI 0.01) or EV71 (MOI 0.01). EV71 was examined in miRNA-transfected cells, but was not used as part of the entire library screen.

We investigated whether miRNA-transfection with miR-134 inhibited EV71 replication. Mimics/ inhibitors against the C. elegans miR-67 were used as non-targeting controls while siRNAs targeting the PV capsid (siPol) were used as positive control. Twenty-four hours post-infection, cells were stored at $-80^{\circ} \mathrm{C}$. miRNA effects on Sabin-2 replication were evaluated by a PV type-II specific ELISA ${ }^{14,15}$. Absorbance values were normalized to non-targeting miRNA mimic controls and converted to a $\mathrm{z}$-score. Mimics that resulted in a $\mathrm{z}$-score $\leq-1.5$ s.d. were deemed hits and subjected to validation experiments which utilized miRNA inhibitors targeting the same genes (Table 1). Several miRNAs suppressed PV replication. MiR-134 and miR-138 had the most potent antiviral effect against PV Sabin-2 following transfection (Fig. 1). For this reason, the antiviral effects of miR-134 were evaluated further and the resulting data is outlined here. MiR-138 is currently still under evaluation. Ingenuity pathway analysis (IPA; QIAGEN) was utilized to evaluate protein networks associated with PV replication to predict targets of miR-134 that may be involved in PV replication. TargetScan analysis was used to predict target transcripts via seed region complementarity. IPA and TargetScan analysis revealed many predicted targets, of interest was the nuclear transport system protein (RAN) because it miR-197 inhibited EV72 replication ${ }^{16}$. We confirmed by qPCR of RAN mRNA and miR-134 transcript levels following miR-mimic transfection (Fig. 2). To confirm RAN's role in PV replication, RAN was silenced via siRNA i prior to infection with Sabin-2 PV. Silencing inhibited PV replication in both cell lines (Fig. 3). miR-134 also drastically reduced EV71 replication (Fig. 4). It is also possible that miR-134 may regulate other host genes required for PV replication, which is supported by the evidence that one miRNA can regulate large numbers of target mRNAs ${ }^{17}$. Hits from primary screen were validated using miRNA specific inhibitors. These findings demonstrate miRNA regulation of PV and EV71 and may provide a novel approach in combating infection.

\section{Methods}

\section{Cell culture and viruses}

HEp-2 is an adherent human epidermoid carcinoma cell line that support PV replication. Caco-2 cells, a colorectal adenocarcinoma (HTB-37) adherent epithelial cell line derived from human colon tissue, were used in conjunction with HEp-2 cells to evaluate the antiviral effects of miR-134 against PV replication. Effects of target gene silencing on poliovirus replication can be detected $24-48 \mathrm{~h}$ post-infection (pi), and were utilized for the miRNA screen. Human muscle rhabdomyosarcoma (RD) cells are an adherent cell which supports enterovirus 71 (EV 71) replication and are routinely utilized to determine enterovirus titers post-miRNA transfection. All cell lines were cultured under the same conditions. All experiments were completed with cells at the same passage. As part of this study we investigated whether miRNA-transfection with miR-134 also inhibited replication of EV71, a familial relative of PV, in an effort to evaluate whether viruses within the same family exhibiting similar replication pathways would also be affected by miR-134. These experiments utilized the same negative control miRNA and siRNA as the PV evaluation ${ }^{18}$. 


\begin{tabular}{|c|c|c|c|c|c|c|c|c|}
\hline miRNA & PreCursor Accession & Normalize Z-score & miRNA & PreCursor Accession & Normalize Z-score & miRNA & PreCursor Accession & Normalize Z-score \\
\hline hsa-let-7i ${ }^{*}$ & MI0000434 & -1.6437 & hsa-miR-29b & MI0000105 & -2.0564 & hsa-miR-432 & MI0003133 & -1.6267 \\
\hline hsa-miR-1181 & MI0006274 & -1.5697 & hsa-miR-29c & MI0000735 & -1.8369 & hsa-mir-4326 & MIMAT0016888 & -1.5294 \\
\hline hsa-miR-1182 & MI0006275 & -1.7367 & hsa-miR-301b & MI0005568 & -1.7121 & hsa-miR-450b-3p & MI0005531 & -1.6297 \\
\hline hsa-miR-1207-5p & MI0006340 & -1.7136 & hsa-miR-30c & MI0000736 & -1.5034 & hsa-miR-483-5p & MI0002467 & -1.8311 \\
\hline hsa-miR-1227 & MI0006316 & -1.821 & hsa-mir-3118-1 & MIMAT0014980 & -1.8229 & hsa-miR-491-5p & MI0003126 & -1.8349 \\
\hline hsa-mir-1244-2 & MI0015974 & -1.6289 & hsa-mir-3123 & MIMAT0014985 & -1.5285 & hsa-miR-497 & MI0003138 & -1.5982 \\
\hline hsa-miR-1250 & MI0006385 & -2.173 & hsa-mir-3127 & MI0014144 & -1.9938 & hsa-miR-509-3p & MI0005717 & -2.185 \\
\hline hsa-miR-1255b & MI0006435 & -1.7509 & hsa-mir-3132 & MIMAT0014997 & -2.0313 & hsa-miR-516b & MI0003172 & -1.7892 \\
\hline hsa-miR-1257 & MI0006391 & -2.0261 & hsa-mir-3140 & MI0014163 & -1.8811 & hsa-miR-517b & MI0003165 & -1.5992 \\
\hline hsa-miR-125b & MI0000446 & -1.7329 & hsa-mir-3175 & MIMAT0015052 & -1.5215 & hsa-miR-518a-5p & MI0003170 & -1.5166 \\
\hline hsa-miR-1274a & MI0006410 & -1.5221 & hsa-mir-3186 & MI0014229 & -1.7061 & hsa-miR-518e & MI0003169 & -1.605 \\
\hline hsa-miR-1275 & MI0006415 & -1.8306 & hsa-mir-3190 & MIMAT0015073 & -1.7578 & hsa-miR-522 & MI0003177 & -1.8432 \\
\hline hsa-miR-129-3p & MI0000473 & -1.7159 & hsa-mir-3191 & MI0014236 & -1.6572 & hsa-miR-523 & MI0003153 & -1.617 \\
\hline hsa-miR-129* & MI0000252 & -1.8363 & hsa-mir-3192 & MIMAT0015076 & -2.0036 & hsa-miR-544 & MI0003515 & -1.6514 \\
\hline hsa-miR-1293 & MI0006355 & -1.7672 & hsa-mir-3194 & MI0014239 & -1.721 & hsa-miR-545* & MI0003516 & -1.8565 \\
\hline hsa-miR-1298 & MI0003938 & -1.5262 & hsa-miR-320d & MI0008190 & -1.5835 & hsa-mir-548aa-1 & MIMAT0018447 & -1.9087 \\
\hline hsa-miR-130a & MI0000448 & -1.6326 & hsa-miR-323-5p & MI0000807 & $\begin{array}{l}-1.8961 \\
\end{array}$ & hsa-miR-548n & MI0006399 & -1.5612 \\
\hline hsa-miR-134 & MI0000474 & -2.3096 & hsa-miR-324-5p & MI0000813 & -1.5969 & hsa-miR-555 & MI0003561 & -2.0986 \\
\hline hsa-miR-138 & MI0000476 & -2.4403 & hsa-miR-330-5p & MI0000803 & -1.8413 & hsa-miR-588 & MI0003597 & -1.677 \\
\hline hsa-miR-149* & MI0000478 & -1.6051 & hsa-miR-338-5p & MI0000814 & -1.8383 & hsa-miR-597 & MI0003609 & -1.5576 \\
\hline hsa-miR-15b* & MI0000438 & -1.6419 & hsa-miR-342-5p & MI0000805 & -1.898 & hsa-miR-608 & MI0003621 & -1.744 \\
\hline hsa-miR-16 & MI0000070 & -1.9572 & hsa-miR-362-5p & MI0000762 & -1.5916 & hsa-miR-609 & MI0003622 & -1.7592 \\
\hline hsa-miR-1908 & MI0008329 & -1.5895 & hsa-miR-363 & MI0000764 & -1.6293 & hsa-miR-613 & MI0003626 & -1.7465 \\
\hline hsa-miR-1909 & MI0008330 & -1.8725 & hsa-mir-3661 & MIMAT0018082 & -1.7807 & hsa-miR-622 & MI0003636 & -2.0702 \\
\hline hsa-miR-1914* & MI0008335 & -1.6261 & hsa-mir-3691 & MI0016092 & -1.5575 & hsa-miR-624 & MI0003638 & -1.6856 \\
\hline hsa-miR-195 & MI0000489 & -1.7648 & hsa-miR-381 & MI0000789 & -1.6366 & hsa-miR-628-5p & MI0003642 & -1.8112 \\
\hline hsa-miR-197 & MI0000239 & -1.8252 & hsa-mir-3915 & MIMAT0018189 & -1.5228 & hsa-miR-630 & MI0003644 & -1.8071 \\
\hline hsa-miR-218-2* & MI0000295 & -1.5057 & hsa-miR-411* & MI0003675 & -1.7965 & hsa-miR-663 & MI0003672 & -1.6806 \\
\hline hsa-miR-220c & MI0005536 & -1.6914 & hsa-miR-421 & MI0003685 & -1.7236 & hsa-mir-676 & MI0016436 & -1.5983 \\
\hline hsa-miR-224* & MI0000301 & -1.8876 & hsa-miR-424 & MI0001446 & -1.7503 & hsa-miR-7-2* & MI0000264 & -2.0278 \\
\hline hsa-mir-2278 & MIMAT0011778 & -1.5577 & hsa-mir- 4252 & MIMAT0016886 & -1.5233 & hsa-miR-768-3p & MI0005117 & -1.6025 \\
\hline hsa-mir-2355 & MI0015873 & -1.5942 & hsa-mir- 4255 & MIMAT0016885 & -1.5652 & hsa-miR-769-3p & MI0003834 & -1.977 \\
\hline hsa-miR-26a & MI0000083 & -1.6853 & hsa-mir-4265 & MIMAT0016891 & -1.6974 & hsa-miR-801 & MI0005202 & -1.561 \\
\hline hsa-miR-26b & MI0000084 & -1.7231 & hsa-mir-4270 & MIMAT0016900 & -1.5457 & hsa-miR-922 & MI0005714 & -1.5015 \\
\hline hsa-miR-27a* & MI0000085 & -1.9841 & hsa-mir-4292 & MIMAT0016919 & -1.5023 & hsa-miR-924 & MI0005716 & -1.5341 \\
\hline hsa-miR-29a & MI0000087 & -1.8126 & hsa-miR-431* & MIMAT0004757 & -1.8885 & hsa-miR-92a-1* & MI0000093 & -1.5144 \\
\hline
\end{tabular}

Table 1. Top anti-PV miRNA hits. Listed are the top 108 miRNA $(z$-score $\leq-1.5)$ which reduced Sabin-2 replication following transfection.

Briefly, cells were cultured in Dulbecco's modified eagle medium (DMEM; Hyclone) supplemented with $10 \%$ heat-inactivated fetal bovine serum (FBS) under cell culture conditions $\left(37^{\circ} \mathrm{C}, 5 \% \mathrm{CO}_{2}\right)$. Cells were passaged $24 \mathrm{~h}$ prior to transfection. Briefly, cell culture media was removed and cells washed with 1X PBS to remove excess media and FBS. Following washing, cells were detached from the culture flask via addition and incubation with $0.05 \%$ Trypsin. Cells were incubated with trypsin under cell culture conditions until cells detached from the flask surface. Trypsin was neutralized with equal volumes of cell culture media containing 10\% FBS. Trypsonized cells were removed from the cell culture flask and transferred to a $50 \mathrm{ml}$ conical tube. Cell suspension was pelleted via centrifugation $(1 \mathrm{~min}$ at $1,200 \times \mathrm{g})$. Supernatant was discarded and cells were resuspended in cell culture media containing 10\% FBS by pipetting. Resuspended cells were then transferred back to the cell culture flask at the appropriate concentration and incubated under cell culture conditions. Master cell stocks were cultivated and stored at $-80^{\circ} \mathrm{C}$. Passage numbers were kept consistent throughout all experiments to reduce variability. HEp-2Cand Caco-2 cells were utilized at P164 and P46, respectively.

The Sabin-2 PV vaccine strain was used throughout this study. This strain of PV is propagated in the African Green Monkey Kidney cell line Vero-P, the approved cell line for polio vaccine production. Cell 
a

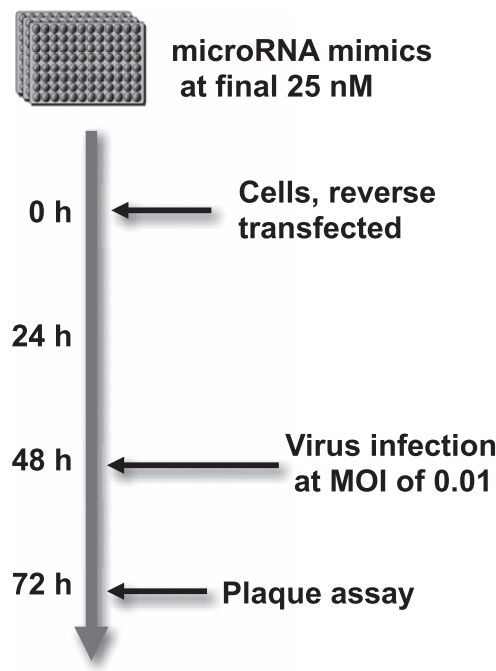

b
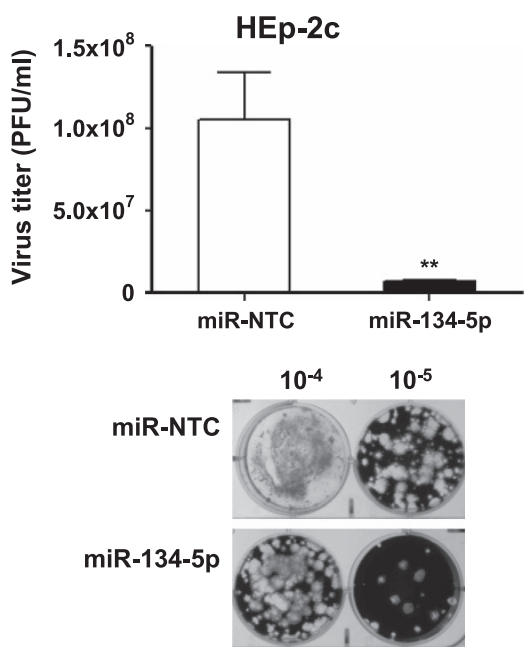

c
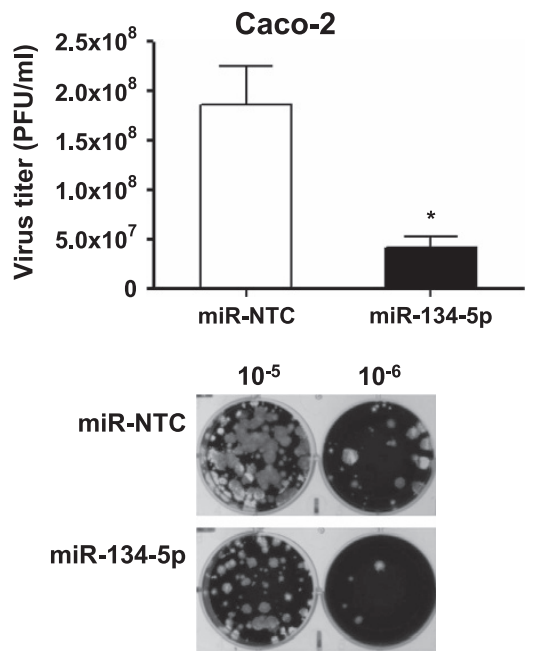

Figure 1. miR-134 inhibits sabin-2 replication in HEp-2c and Caco-2 cells. (a) Schematic of the experimental design. To evaluate if antiviral effect of miR-134 is cell type-specific, HEp-2c (b) and Caco-2 (c) cells were reverse-transfected with miR-134-5p mimic or miR mimic non-targeting scrambled negative control (miR-NTC) at a final concentration of $25 \mathrm{nM}$. After $48 \mathrm{~h}$, the transfected cells were infected with Sabin-2 at MOI of 0.01 for $24 \mathrm{~h}$ and the virus titers were determined by plaque assay. Error bars represent s.e.m. and the significant differences are compared with miR-control. Each experiment was performed in quadruplicate and each experiment was repeated twice. The results were reproduced and the data in the figure were representative of 8 plates. ${ }^{\star} P<0.05,{ }^{* *} P<0.01$.

a

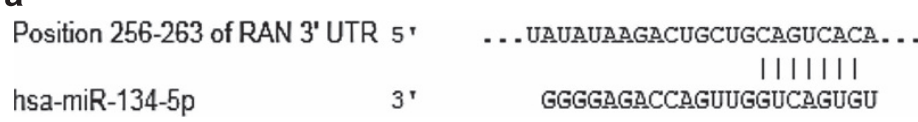

b

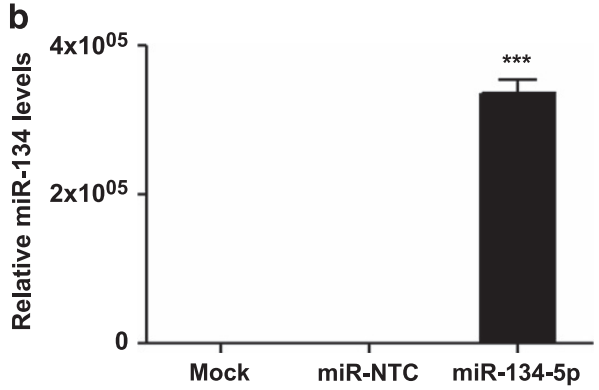

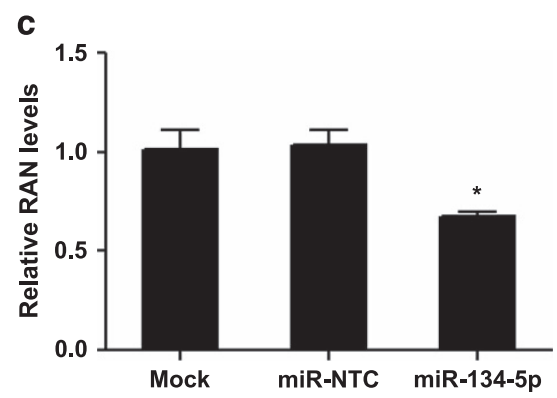

Figure 2. RAN is a target of miR-134. (a) Sequence of the putative binding site between miR-134 and 3'UTR of RAN. (b) and (c) Hep-2c cells were reverse-transfected with miR-134-5p or miR mimic nontargeting scrambled negative control (miR-NTC) at a final concentration of $25 \mathrm{nM}$. After $48 \mathrm{~h}$, total RNA was isolated from HEp-2c cells, and then miR-134 (b) and RAN (c) mRNA levels were determined by qRT-PCR. The data were normalized to the $18 \mathrm{~S}$ rRNA gene and were expressed as fold change relative to mocktransfected cells. The significant differences are compared with miR-control. Each experiment was performed in quadruplicate and each experiment was repeated twice. The results were reproduced and the data in the figure were representative of 8 plates. Error bars represent s.e.m. ${ }^{\star} P<0.05,{ }^{* * *} P<0.001$.

stocks were obtained from the Centers of Disease Control and Prevention (CDC, Atlanta) who in turn obtained stocks from the Serum Institute of India (SI). Vero-P stocks were cultured under cell culture conditions at $37^{\circ} \mathrm{C}, 5 \% \mathrm{CO}_{2}$ in Dulbecco's modified Eagle's medium (DMEM; Hyclone, GE Healthcare) supplemented with $10 \%$ heat inactivated fetal bovine serum (FBS; Hyclone, GE Healthcare). Master cell stocks were cultivated and stored at $-80^{\circ} \mathrm{C}$. Passage number were kept consistent throughout all 
a

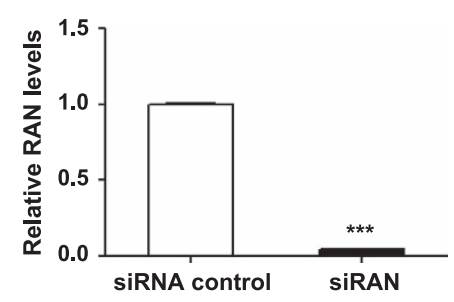

b

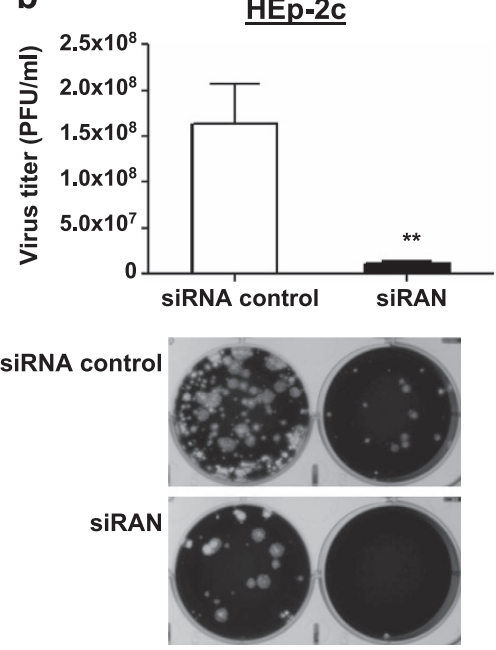

c

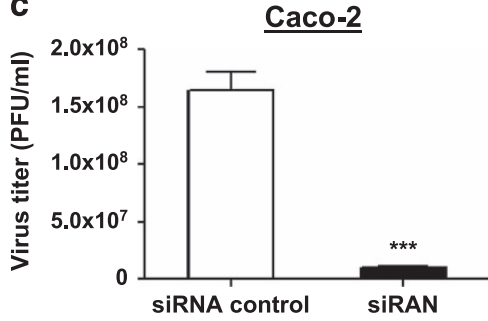

SiRNA control

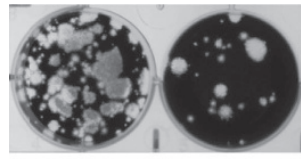

SIRAN

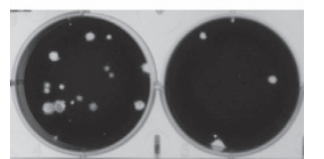

Figure 3. Silencing of RAN inhibits PV replication in HEp-2c and Caco-2 cells. The cells were reversetransfected with siRAN or siRNA control at a final concentration of $25 \mathrm{nM}$. After $48 \mathrm{~h}$, (a) total RNA was isolated from HEp-2c cells and RAN mRNA level was determined by qRT-PCR. The data were normalized to the $18 \mathrm{~S}$ rRNA gene and were expressed as fold change relative to siRNA control. The transfected Hep-2c (b) and Caco-2 (c) cells were infected with Sabin-2 strain at an MOI of 0.01 for $24 \mathrm{~h}$, and then the virus titers were determined by plaque assay. The significant differences are compared with siRNA control. Error bars represent s.e.m. of quadruplicate. Each experiment was performed in quadruplicate and each experiment was repeated twice. The results were reproduced and the data in the figure were representative of 8 plates. ${ }^{* *} P<0.01,{ }^{* * *} P<0.001$.
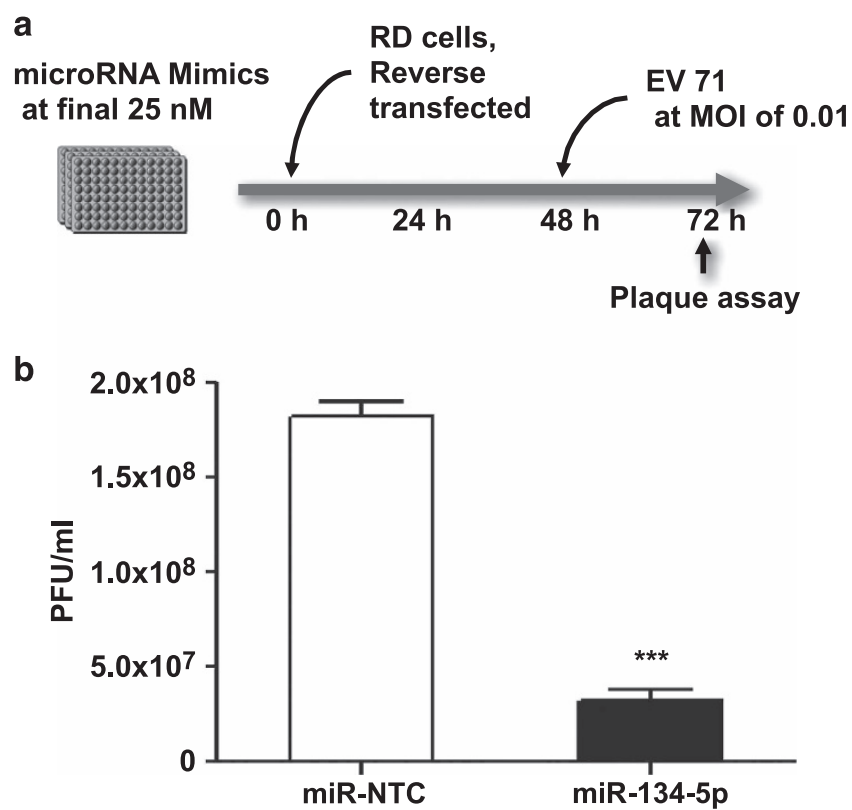

Figure 4. miR-134 targets EV71 replication in RD cells. (a) Schematic of the experimental design.

(b) RD cells were reverse-transfected with miR-134-5p or miR mimic non-targeting scrambled negative control (miR-NTC) at final concentration of $25 \mathrm{nM}$. $48 \mathrm{~h}$ later the cells were infected with EV71 at MOI of 0.01 for $24 \mathrm{~h}$ and the antiviral effect of miR-134 was determined by plaque assay. Error bars represent s.e.m. of of quadruplicate. Each experiment was performed in quadruplicate and each experiment was repeated twice. The results were reproduced and the data in the figure were representative of 8 plates. The significant differences are compared with miR-control. ${ }^{* *} P<0.001$. 
experiments to reduce variability. Viral stocks were cultivated, viral titers determined by plaque assay and $50 \%$ cell culture infectious dose $\left(\mathrm{CCID}_{50}\right)$, aliquoted and stored at $-80^{\circ} \mathrm{C}$ until use $\mathrm{s}^{19}$.

\section{Primary screening and miRNA mimic transfection}

The primary miRNA mimic screen included a library of 1,208 miRNA mimics (GE Dharmacon-Thermo Scientific; CS-001010 Human Mimics Lot 10100 and CS-001015 Supplement Human Mimic 16.0 Lot 11144). Screening was completed in triplicate using a 96-well plate format with HEp-2C cells $\left(10^{3}\right.$ cells per well). HEp-2C cells were reverse transfected to a final mimic concentration of $25 \mathrm{nM}$. The Dharmacon miRIDIAN microRNA mimic negative control (miR-NTC; CN-001000-01) was used as a negative control and a siRNA targeting the poliovirus capsid-coding region (siPol) (CDC, provided by Dr Steve Oberste) was utilized to monitor silencing effects on poliovirus replication in all assays. A cytoxicity transfection control siRNA (siTOX) (GE Healthcare Dharmacon TOX Transfection Control, Cat. \# D0015000120) was utilized to visually confirm effective productive transfection. Cells successfully transfected with siTOX undergo apoptosis resulting in cell death. Briefly, mimics were incubated at room temperature for $20 \mathrm{~min}$ in $0.3 \%$ ( $0.3 \mathrm{ul}$ per well) of DharmaFECT transfection reagent 4 (DF4) (Dharmacon-Thermo Scientific) prepared in serum-free medium (Opti-MEM, Invitrogen Inc.) prior to addition of cells in Dulbecco's modified Eagle's medium (DMEM, Invitrogen) supplemented with $10 \%$ calf serum. Cells were cultured for $48 \mathrm{~h}$ at $37^{\circ} \mathrm{C}, 5 \% \mathrm{CO}_{2}$. Forty-eight hours post-transfection, media was removed, cells were washed with phosphate buffer saline (PBS; Hyclone, GE Healthcare) and infected with Sabin 2 at an MOI $=0.01$ Virus was diluted in DMEM containing $2 \%$ calf serum and $1 \%$ penicillinstreptomycin. Twenty-four hours post-infection, HEp-2C cells were removed from the culture incubator and stored at $-80^{\circ} \mathrm{C}$ until evaluated.

Validation screening and removal of false positives

Following the primary miRNA screen, in an effort to reduce false positives relative to transfection variability, individual miRNA inhibitors (GE Dharmacon Thermo-Fisher; IH-001010 Human Inhibitor Lot 09168 and IH-001015 Supplement Human Hairpin Inhibitor Library 16.0) were utilized to validate miRNA hits produced as part of the primary miRNA mimic screen. Hits producing an opposite phenotype as compared to the miRNA mimics screen were taken into future studies. The miRNA mimics are the focus of this study; therefore, the brief screening of the miRNA inhibitors was not included here and only used as a form of phenotype validation. Briefly, individual miRNA inhibitors corresponding to miRNA hits produced as part of the primary mimic screen were reverse-transfected into HEp-2 cells as previously described. At $48 \mathrm{~h}$ post-transfection, cells were infected with Sabin-1, Sabin-2 or Sabin-3 strains $(\mathrm{MOI}=0.01)$. Cells were incubated and replication assessed as previously stated. Messenger RNA levels for each gene were evaluated following transfection by qPCR.

Validation screens were completed to further confirm the anti-virus effects of the identified miRNA mimics using ELISA assay. For the list of 108 anti-polio miRNA mimics identified from the primary miRNA mimics screening (Table 1), we compared the phenotypes with the miRNA inhibitor data. Following miRNA inhibitor experiments, hits that did not result in opposing phenotypes when compared to their mimic counterparts were excluded. The miRNA mimics which lead to cell death were also excluded. We also identified 29 miRNA mimics that when transfected into cells lead to decreased poliovirus replication (Fig. 5). From this data, miRNA-134 was selected for further investigation in this study.

\section{Polio Type II specific antibody-capture ELISA}

A PV type-II specific ELISA was used to determine miRNA effects on Sabin-2 replication. Antibodycapture ELISA for detection of poliovirus was described previously ${ }^{14,15}$. Briefly, $2 \mathrm{HB}$ high-binding 96 -well plates (NUNC; 1424561-PK) were coated with $50 \mathrm{ul}$ of the PV type II specific monoclonal antibody

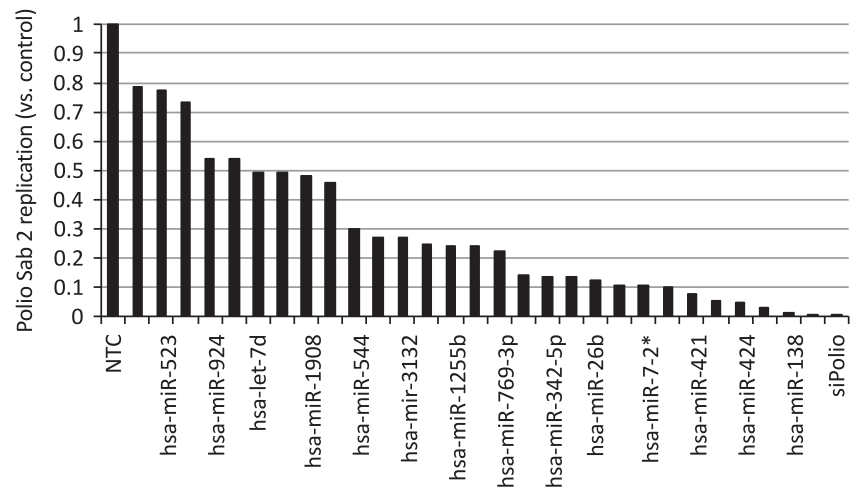

Figure 5. miRNAs inhibit sabin-2 replication in HEp-2c cells. Cells were reverse-transfected with miRNA mimic or miR-control mimic (miR-control) at a final concentration of $25 \mathrm{nM}$. After $48 \mathrm{~h}$, the transfected cells were infected with Sabin-2 at MOI of 0.01 for $24 \mathrm{~h}$ and the virus titers were determined by ESLIA assay. 
(HYB294-06, mouse; Thermo Scientific Pierce) diluted in carbonate-bicarbonate buffer (pH 9.6). Plates were incubated for $16 \mathrm{~h}$ (overnight) at $4{ }^{\circ} \mathrm{C}$ in a moist chamber. Antibody-coated plates were washed with 0.05\% phosphate buffered saline with Tween 20 (PBST). Following washing, plates were blocked with $100 \mu \mathrm{l}$ blocking buffer ( $1 \mathrm{x}$ PBS containing $0.5 \%$ Gelatin and $0.25 \%$ Tween) for $60 \mathrm{~min}$ at $37^{\circ} \mathrm{C}$. Block was removed and plates were washed $4 \mathrm{x}$ with PBST. Fifty microliters of cell lysate was transferred from the thawed infected transfection plate to the antibody-coated plates. Inoculated plates were incubated for $60 \mathrm{~min}$ at $37^{\circ} \mathrm{C}$ in a moist chamber. Subsequently, plates were washed $4 \mathrm{x}$ with PBST and $50 \mathrm{ul}$ of HRP-substrate solution (SureBlue TMB) was added and incubated for $15 \mathrm{~min}$ at $37^{\circ} \mathrm{C}$. The reaction was halted by the addition of $50 \mathrm{ul} \mathrm{H}_{2} \mathrm{SO}_{4}$ stop solution. Absorbance was evaluated at a wavelength of $450 \mathrm{~nm}$ with a plate spectrophotometer (Tecan). Absorbance values were normalized to non-targeting miRNA mimic controls and converted to a $\mathrm{Z}$-score. Z-scores were used to determine whether hits are positive according to methods describe previously ${ }^{20}$. Hits with a $\mathrm{Z}$-score $\leq-1.5$ s.d. were subjected to validation experiments in HEp-2C cells.

\section{Poliovirus (PV) and enterovirus 71 (EV71) plaque assays}

PV and EV71 viral titers were determined post-transfection by either a HEp-2 or RD cell-based plaque assay, respectively ${ }^{19,21}$. Briefly, $1.2 \times 10^{6}$ cells were seeded in each well of six well plates and incubated overnight under standard cell culture conditions. The next day culture media was removed and cells were washed with minimal essential medium (MEM; Hyclone, GE Healthcare, USA) supplemented with $2 \%$ fetal bovine serum (FBS). Next, samples were serially diluted 10 -fold in MEM with $2 \%$ FBS and added to washed plates. Plates were incubated at $37^{\circ} \mathrm{C}, 5 \% \mathrm{CO}_{2}$ for $1 \mathrm{~h}$. The inoculum was removed and plates were overlaid with $0.9 \%$ nutrient agarose gel in MEM supplemented with $2 \%$ FBS, followed by incubation in a $37^{\circ} \mathrm{C}$ incubator with $5 \% \mathrm{CO}_{2}$ for 3 days. Agarose overlay was removed and cells fixed with $10 \%$ formaldehyde in PBS. Plaques were visualized by staining with crystal violet. Virus titers were expressed as PFU $\mathrm{ml}^{-1}$.

\section{RNA isolation and qRT-PCR}

Cells were homogenized and total RNA was extracted using RNAzol RT reagent (Molecular Research Center) according to the manufacturer's instruction. The concentration of the purified RNA was determined by NanoDrop spectrophotometer (Thermo Scientific). To examine mature miR-134-5 expression, $300 \mathrm{ng}$ total RNA was reverse-transcribed to cDNA using the miRNA 1st-Strand cDNA synthesis kit (Agilent Technologies) according to the manufacturer's protocol. For the RAN gene, $300 \mathrm{ng}$ total RNA was reverse-transcribed to cDNA using the AffinityScript QPCR cDNA synthesis kit (Agilent Technologies). For the quantification of individual genes, cDNAs were diluted in water and quantified using Brilliant III Ultra-Fast SYBR Green QPCR Master Mix (Agilent Technologies) in an Agilent Mx3005P instrument. Primers specific for miR-134-5p (forward, $5^{\prime}$-TGTGACTGGTTGA CCA-3'; reverse, universal primer) RAN (forward, 5'-GAAGCTCATTGGAGACCCTAAC-3'; reverse, $5^{\prime}$-CAACCTCTAAGTCGTGCTCATAC-3') were used. The gene expression levels were normalized to 18 s rRNA gene as an internal control.

\section{Ingenuity pathway analysis and TargetScan analysis}

QIAGEN's Ingenuity pathway analysis (IPA; QIAGEN Redwood City; www.qiagen.com/ingenuity) was utilized to evaluate protein networks associated with PV replication in an effort to predict targets of miR-134 that may be involved in poliovirus infection and replication. TargetScan analysis (Whitehead Institute for Biomedical Research; http://www.targetscan.org/) was used to predict target transcripts via seed region complementarity. Pathway analysis and TargetScan analysis revealed many predicted targets, of interest was the nuclear transport system protein (RAN) because it has been previously published that another microRNA, miR-197, inhibited enterovirus 71 replication by targeting the RAN protein ${ }^{16}$.

\section{Data normalization and statistical analysis}

The current screen was examined using $Z^{\prime}$-factor ( $1>Z^{\prime}>0.5$ : Excellent assay; 0.5 $>Z^{\prime}>0$ : acceptable) to evaluate its quality. The majority of our screen fell into a $Z^{\prime}$ factor $\left(0.5<Z^{\prime}<1.0\right)$ which reflects high-quality of the primary screen. Afterwards, the primary screen data was normalized to the entire plate and generated the mean $(\mu)$ of the data to zero and the standard deviation (s.d.) to 1. To achieve a high-quality and robust screen, we used siTOX (GE Healthcare Dharmacon TOX ${ }^{\mathrm{s}}$ Transfection Control, Cat. \# D0015000120) to monitor the transfection efficiency; we also used a siRNA targeting poliovirus (CDC, provided by Dr Steve Oberste) to monitor silencing effects on poliovirus replication (see transfection control section). A non-targeting siRNA (GE Healthcare Dharmacon: Cat. \# D-001810-0X) used as a negative control. All experiments were performed in at least two independent replicates with each experiment consisting of duplicates for each miRNA targeting a specific mRNA transcript for the gene of interest. ELISA absorbance values were normalized by correcting the raw data. The mean of the replicates where calculated and standardized using Z-score analysis. The mean of the wells treated with experimental miRNAs for each miRNA were compared to the mean of negative control treated wells. The Z-score was calculated by finding the difference between the normalized score and the mean of the population and dividing this by the standard deviation of the population. Positive hits from the primary screen are scored by Z-score $>1.5$ s.d. 


\begin{tabular}{|l|l|l|l|l|l|}
\hline miRNA & PreCursor Accession & Normalized Z-score & \multicolumn{1}{|c|}{ miRNA } & PreCursor Accession & Normalized Z-score \\
\hline hsa-miR-106a & MI0000113 & 2.5029 & hsa-mir-3622b & MI0016014 & 2.8955 \\
\hline hsa-miR-1183 & MI0006276 & 2.2776 & hsa-mir-3650 & MIMAT0018070 & 3.0868 \\
\hline hsa-miR-1256 & MI0006390 & 2.2043 & hsa-miR-371-3p & MIMAT0000723 & 3.0328 \\
\hline hsa-mir-1270-2 & MI0006407 & 1.8131 & hsa-mir-3913-1 & MI0016417 & 1.8771 \\
\hline hsa-miR-1290 & MI0006352 & 2.1255 & hsa-miR-411 & MI0003675 & 1.6903 \\
\hline hsa-miR-148b & MI0000811 & 2.1563 & hsa-miR-429 & MIMAT0001536 & 2.3367 \\
\hline hsa-miR-182 & MI0000272 & 1.813 & hsa-mir-4300 & MIMAT0016853 & 2.2905 \\
\hline hsa-miR-1825 & MI0008193 & 1.6288 & hsa-mir-4311 & MIMAT0016863 & 1.8049 \\
\hline hsa-miR-185* & MI0000482 & 2.5167 & hsa-miR-488 & MI0003123 & 1.7722 \\
\hline hsa-miR-190b & MI0005545 & 1.5325 & hsa-miR-512-3p & MI0003140 & 2.4828 \\
\hline hsa-miR-1977 & MI0009987 & 2.8377 & hsa-miR-513a-5p & MI0003191 & 1.6767 \\
\hline hsa-miR-1978 & MI0009988 & 2.033 & hsa-miR-519c-3p & MI0003148 & 1.5004 \\
\hline hsa-mir-2115* & MIMAT0011159 & 2.2428 & hsa-miR-519d & MI0003162 & 1.789 \\
\hline hsa-miR-216a & MI0000292 & 1.5712 & hsa-miR-520c-3p & MI0003158 & 2.1928 \\
\hline hsa-miR-22 & MI0000078 & 1.6283 & hsa-miR-520d-3p & MI0003164 & 2.3981 \\
\hline hsa-miR-221* & MI0000298 & 2.2304 & hsa-miR-520e & MI0003143 & 1.7425 \\
\hline hsa-mir-3155 & MIMAT0015029 & 1.7369 & hsa-miR-526b* & MI0003150 & 2.2129 \\
\hline hsa-mir-3160-1 & MI0014189 & 2.0883 & hsa-miR-559 & MI0003565 & 1.8168 \\
\hline hsa-mir-3162 & MI0014192 & 2.7709 & hsa-miR-571 & MI0003578 & 1.9588 \\
\hline hsa-mir-3187 & MI0014231 & 2.0815 & hsa-miR-576-3p & MI0003583 & 2.4894 \\
\hline hsa-mir-3605 & MI0015995 & 1.7167 & hsa-miR-589 & MI0003599 & 2.0177 \\
\hline hsa-miR-361-5p & MI0000760 & 2.295 & hsa-miR-598 & MI0003610 & 1.5474 \\
\hline hsa-mir-3620 & MI0016011 & 2.5204 & hsa-miR-9 & MI0000466 & 2.1298 \\
\hline
\end{tabular}

Table 2. Top pro-PV miRNA hits. Listed are the top 46 miRNA ( $z$-score $\geq 1.5$ ) which increased Sabin-2 replication following transfection.

All results are presented as means \pm s.e. Statistical analysis was done using the one-way ANOVA with Turkey's multiple comparison at $95 \%$ confidence level. $P$-values $<0.05$ were considered statistically significant.

\section{Data Records}

\section{Data record 1}

The miRNA mimic screen data presented is available at PubChem under NCBI PubChem BioAssay AID 1224906 (Data Citation 1). In this RNAi screen, miRNA mimics corresponding to 1,208 known miRNAs in the human genome were screened in HEp-2 cells for their regulatory effects against Sabin-2 poliovirus. One hundred eight miRNAs resulted in a substantial reduction of poliovirus replication relative to non-targeting control ( $\mathrm{z}$-scores $\leq-1.5)$. Forty-six miRNAs resulted in a substantial increase of poliovirus replication relative to non-targeting control ( $\mathrm{z}$-scores $\geq 1.5$ ) (Table 2). Included within this dataset are the hits which resulted in a standard $\mathrm{z}$-score $\leq-0.5$ and $\mathrm{z}$-score $\geq 0.5$ compared to non-targeting control.

\section{Data record 2}

The miRNA inhibitor data is available at PubChem under NCBI PubChem BioAssay AID 1224851 (Data Citation 2). The top miRNA hits from the mimic screening assays were re-screened in HEp-2 cells, to eliminate false-positive hits. In this case, miRNA inhibitors were tested to determine if they induced a phenotype which correlated with that of the miRNA mimic results. Included within this dataset are the z-scores of all miRNAs inhibitors tested.

\section{Technical Validation}

\section{Transfection optimization}

To establish a highly efficient miRNA transfection protocol in a high-throughput screening (HTS) fashion, we first investigated four individual DharmaFECT transfection reagents (DF1-4). We identified that DF4 is the most effective in gene silencing for both HEp-2. Further we performed the titration of the concentration of DF4 in both cell lines. In the case with $0.3 \%$ DF4 concentration, the GAPDH silencing was achieved $>90 \%$, and cell toxicity was not observed in either microscopy examination or MTT assay. 

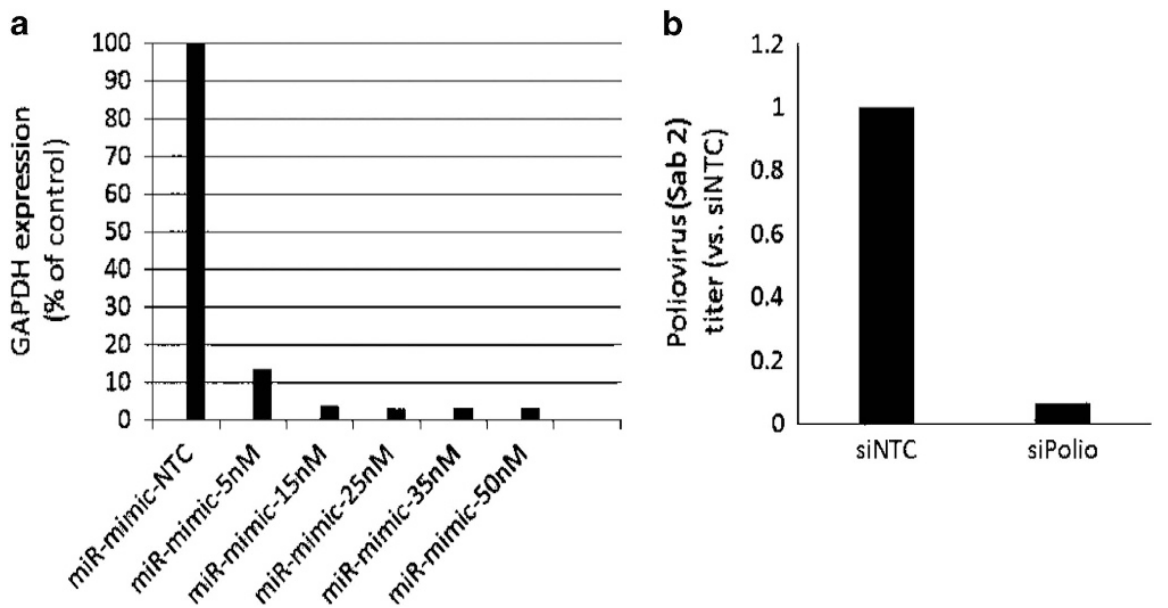

Figure 6. (a) optimization of transfection using miRIDIAN microRNA mimic housekeeping positive control \#2 (GAPDH) (GE Dharmacon; Cat \# CP-001000-02). (b) Optimization of positive control (siPol) for virus replication (CDC, provided by Dr Steve Oberste).

\section{Cytotoxicity assays}

Cytotoxicity as a result of miRNA transfection was evaluated by CytoTox-Glo assay (Promega) according to manufacturer instructions. Briefly, HEp-2 cells were reverse transfected with miRNA mimics as described previously ${ }^{22}$. Forty-eight hours post-transfection cellular toxicity was evaluated. Values were normalized to siTOX control (100\% cytotoxic). MiRNAs which elicited greater than $20 \%$ toxicity were excluded as is standard practice. In this study, we focused on the miRNA mimics screening, and observed some miR-induced cell death. Toxic miRNAs that lead to cell death including miR-1275, miR-129-3P, miR509, miR-1909, miR323-5P, miR-4265, miR-3661 were excluded from further studies. For the list of hits identified from miRNA mimics screening, we did not observe significant cell death for the corresponding miRNA inhibitors.

\section{Transfection controls}

A high-quality RNAi screen integrates both excellent experimental design and computational strategies for quality control. A clear distinction between a positive control and a negative reference is paramount. In the current miRNA screen in HEp-2 cell line, a positive control siRNA specifically targeting poliovirus (Poliovirus-specific (VP1 \& 3D) and a negative control (non-targeting siRNA) were clearly distinguished from each other in all 96-well plates transfected with siRNAs. siTOX (GE Healthcare Dharmacon TOX Transfection Control, Cat. \# D0015000120) was used to serve as another indicator for transfection efficiency and a mock control used as background normalization.

As part of developing a screening assay with low variation and a strong signal to background ratio we conducted pilot studies to optimize screening conditions. These conditions included optimizing titration of miRNA and siRNAs used to determine the most effective concentrations. Assay responses to negative and positive miRNA and siRNA controls were also evaluated and optimized to determine baseline values and standard deviation value for the calculation of Z-scores. Z-scores were subsequently used to determine hits for further screening. Transfection efficiency was optimized via transfection with miRIDIAN microRNA Mimic housekeeping positive Control \#2 (GAPDH) (Cat. \# CP-001000-02) as well as the inclusion of siTOX transfection control as previously mentioned. MRNA transcription levels were evaluated by qPCR following miRNA transfection and compared to GAPDH control to confirm miRNA mediated mRNA knockdown. Positive controls for poliovirus screening were carefully selected and optimized (Fig. 6) ${ }^{23}$. The culmination of this data suggests high transfection efficiency, low variability and substantial positive control values concluding that our screening procedure is robust.

\section{References}

1. Guo, J., Bolivar-Wagers, S., Srinivas, N., Holubar, M. \& Maldonado, Y. Immunodeficiency-related vaccine-derived poliovirus (iVDPV) cases: a systematic review and implications for polio eradication. Vaccine 33, 1235-1242 (2015).

2. Minor, P. Vaccine-derived poliovirus (VDPV): Impact on poliomyelitis eradication. Vaccine 27, 2649-2652 (2009).

3. Wong, S. S., Yip, C. C., Lau, S. K. \& Yuen, K. Y. Human enterovirus 71 and hand, foot and mouth disease. Epidemiol Infect 138, 1071-1089 (2010).

4. Liang, Z. \& Wang, J. EV71 vaccine, an invaluable gift for children. Clin Transl Immunology 3, e28 (2014).

5. Bartel, D. P. MicroRNAs: Genomics, Biogenesis, Mechanism, and Function. Cell Press 116, 281-297 (2004).

6. Filipowicz, W., Bhattacharyya, S. N. \& Sonenberg, N. Mechanisms of post-transcriptional regulation by microRNAs: are the answers in sight? Nature reviews. Genetics 9, 102-114 (2008).

7. Skalsky, R. L. \& Cullen, B. R. Viruses, microRNAs, and host interactions. Annual review of microbiology 64, 123-141 (2010).

8. Umbach, J. L. \& Cullen, B. R. The role of RNAi and microRNAs in animal virus replication and antiviral immunity. Genes \& development 23, 1151-1164 (2009). 
9. Wang, X. et al. MicroRNA identification based on sequence and structure alignment. Bioinformatics 21, 3610-3614 (2005).

10. Muller, S. \& Imler, J. L. Dicing with viruses: microRNAs as antiviral factors. Immunity 27, 1-3 (2007).

11. Trobaugh, D. W. et al. RNA viruses can hijack vertebrate microRNAs to suppress innate immunity. Nature 506, 245-248 (2014).

12. Cheng, M. et al. High-Throughput Profiling of Alpha Interferon- and Interleukin-28B- Regulated MicroRNAs and Identification of let-7s with Anti-Hepatitis C Virus Activity by Targeting IGF2BP1. Journal of virology 87, 9707-9718 (2013).

13. Huang, J. Y. et al. MicroRNA-130a can inhibit hepatitis B virus replication via targeting PGClalpha and PPARgamma. Rna 21, 385-400 (2015)

14. Ivanov, A. P. ELISA as a possible alternative to the neutralization test for evaluating the immune response to poliovirus vaccines. Expert Review Vaccines 4, 167-172 (2005).

15. Nibbeling, R. A poliovirus type-specific IgM antibody-capture enzyme-linked immunosorbent assay for the rapid diagnosis of poliomyelitis. Clinical and Diagnostic Virology 2, 113-126 (1994).

16. Tang, W. F. et al. Host MicroRNA miR-197 Plays a Negative Regulatory Role in the Enterovirus 71 Infectious Cycle by Targeting the RAN Protein. Journal of virology 90, 1424-1438 (2015).

17. Lim, L. P. et al. Microarray analysis shows that some microRNAs downregulate large numbers of target mRNAs. Nature 433, 769-773 (2005).

18. Zheng, Z. et al. Human microRNA hsa-miR-296-5p suppresses enterovirus 71 replication by targeting the viral genome. Journal of virology 87, 5645-5656 (2013).

19. Arita, M., Wakita, T. \& Shimizu, H. Characterization of pharmacologically active compounds that inhibit poliovirus and enterovirus 71 infectivity. The Journal of general virology 89, 2518-2530 (2008).

20. Bakre, A. et al. Identification of Host Kinase Genes Required for Influenza Virus Replication and the Regulatory Role of MicroRNAs. PLoS ONE 8, e66796 (2013).

21. Burns, C. C. et al. Modulation of poliovirus replicative fitness in HeLa cells by deoptimization of synonymous codon usage in the capsid region. Journal of virology 80, 3259-3272 (2006).

22. Shim, B. S. et al. MicroRNA-555 has potent antiviral properties against poliovirus. The Journal of general virology $\mathbf{9 7}$, 659-668 (2016).

23. van der Sanden, S. M. et al. Engineering Enhanced Vaccine Cell Lines To Eradicate Vaccine-Preventable Diseases: the Polio End Game. Journal of virology 90, 1694-1704 (2016).

\section{Data Citations}

1. Orr-Burks, N., Shim, B., Wu, W., Bakre, A. \& Tripp, R. NCBI PubChem BioAssay 1224906 (2017).

2. Orr-Burks, N., Shim, B., Wu, W., Bakre, A. \& Tripp, R. NCBI PubChem BioAssay 1224851 (2017).

\section{Acknowledgements}

We would like to thank Dr Steve Oberste, CDC, for providing the poliovirus strains used in these studies. The authors would like to thank the Georgia Research Alliance for supporting the studies.

\section{Author Contributions}

B.-S.S., W.W. and N.O.-B. contributed equally to this study. R.A.T. initiated this project. A.B. contributed to data interpretation and manuscript preparation.

\section{Additional Information}

Competing financial interests: The authors declare no competing financial interests.

How to cite this article: Orr-Burks, N. L. et al. MicroRNA screening identifies miR-134 as a regulator of poliovirus and enterovirus 71 infection. Sci. Data 4:170023 doi: 10.1038/sdata.2017.23 (2017).

Publisher's note: Springer Nature remains neutral with regard to jurisdictional claims in published maps and institutional affiliations.

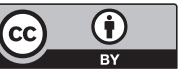

This work is licensed under a Creative Commons Attribution 4.0 International License. The images or other third party material in this article are included in the article's Creative Commons license, unless indicated otherwise in the credit line; if the material is not included under the Creative Commons license, users will need to obtain permission from the license holder to reproduce the material. To view a copy of this license, visit http://creativecommons.org/licenses/by/4.0

Metadata associated with this Data Descriptor is available at http://www.nature.com/sdata/ and is released under the CC0 waiver to maximize reuse.

(c) The Author(s) 2017 